\title{
Shikonin Induced Necroptosis via Reactive Oxygen Species in the T-47D Breast Cancer Cell Line
}

\author{
Zahra Shahsavari $^{1}$, Fatemeh Karami-Tehrani ${ }^{1 *}$, Siamak Salami ${ }^{2}$
}

\begin{abstract}
Breast cancer, the most common cancer in the women, is the leading cause of death. Necrotic signaling pathways will enable targeted therapeutic agents to eliminate apoptosis-resistant cancer cells. In the present study, the effect of shikonin on the induction of cell necroptosis or apoptosis was evaluated using the T-47D breast cancer cell line. The cell death modes, caspase-3 and 8 activities and the levels of reactive oxygen species (ROS) were assessed. Cell death mainly occurred through necroptosis. In the presence of Nec-1, caspase-3 mediated apoptosis was apparent in the shikonin treated cells. Shikonin stimulates ROS generation in the mitochondria of T-47D cells, which causes necroptosis or apoptosis. Induction of necroptosis, as a backup-programmed cell death pathway via ROS stimulation, offers a new strategy for the treatment of breast cancer.
\end{abstract}

Keywords: Shikonin - ROS - necroptosis - apoptosis - breast cancer

Asian Pac J Cancer Prev, 16 (16), 7261-7266

\section{Introduction}

There are several pathways through which a cell death can occur. Apoptosis was the first to be discovered. Initial caspase activation results in a cascade of caspase activity, breaking down cellular components and resulting in cell death. Inhibition of caspase activities protects cells against cytotoxic stimuli and results in necroptosis (Christofferson et al., 2014). While apoptosis has been considered as an original programmed cell death (Salami and KaramiTehrani, 2003; Sadeghi et al., 2015), necroptosis, as a novel type of programmed cell death, is morphologically related to necrosis (Shindo et al., 2013). Necrotic signaling pathways will enable targeted therapeutic agents to eliminate apoptosis-resistant cancer cells (Wang and Zhang, 2015).

In the cell death, TNF receptor will be activated and may be executed through apoptosis or necroptosis (Christofferson DE, 2010 Apr). To induce apoptosis, transition of complex I to cytosolic complex IIa (contains FADD, caspase-8, and RIP1), would occur. When apoptosis is inhibited, necroptosis may be induced by the activation of complex IIb, which requires RIP1K and RIP3K (Christofferson DE, 2010 Apr). The blockers to apoptosis are not problems for necroptosis, since necroptosis is completely different from apoptosis (Xun et al., 2007).

It has been shown that activation of a phosphoglycerate mutase family member 5 or alterations of oxidative status results in ROS production by damaged mitochondria
(Shindo R, 2013).

Drug induced intrinsic ROS results in the cell cytotoxicity, which basically destroys malignant cells or inhibits their proliferation (Yang et al., 2014; Mansoori, 2015). Shikonin, a natural naphthoquinone is a constituent of red pigment extracted from Lithospermum erythrorhizon Siebet Zucc of East Asia. Shikonin and its analogues are strong anticancer agents with weak inducer of resistance to drug (Wu et al., 2013).

Breast cancer, the most common cancer in the women, is the leading cause of death (Siao et al., 2015; Sharifian, 2015; Xin et al., 2015). Therefore, for the treatment of breast cancers, those resistant to the existing therapies, new approaches are required (Ghavami , 2009 ; Singha et al., 2013; Tavakoli-Yaraki et al., 2013; Baghestani et al., 2015; Doval et al., 2015; Meka et al., 2015). Thus in the present study, the effect of shikonin on the induction of cell necroptosis or apoptosis has been evaluated using T-47D breast cancer cell line.

\section{Materials and Methods}

Reagents

Dulbecco's Modified Eagle's Medium/Nutrient F-12 Ham (DMEM/ Ham's F12), Fetal Bovine Serum (FBS) and penicillin-streptomycin were obtained from Gibco (Grand Island, NY, USA) respectively. Shikonin and Nec1 were purchased from CalBiochem (EMD chemicals, Inc. San Diego, CA, USA). Pan Caspase inhibitor, Z-VADFMK, was from BD BioScience (Becton Dickinson, San

${ }^{1}$ Cancer Research Laboratory, Department of Clinical Biochemistry, Faculty of Medical Sciences, Tarbiat Modares University, ${ }^{2}$ Department of Clinical Biochemistry, Faculty of Medicine, Shahid Beheshti University of Medical Sciences, Tehran, Iran *For correspondence: karamitf@modares.ac.ir 
Zahra Shahsavari et al

Jose, CA). The Caspase-3 Fluorometric Assay Kit was bought from Biovision (BioVision, Inc. Milpitas, CA USA). The Casp GLOWTM Fluorescein Active Caspase-8 Staining Kit was from eBioscience (eBioscience, Inc. San Diego, CA USA). Marker GeneTM Live Cell Fluorescent Reactive Oxygen Species Detection Kit was obtained from Marker Gene Technologies (Marker Gene Technologies, Inc. Eugene, Oregon). Other reagents and chemicals were purchased from Sigma Chemical Co (St. Louis, MO, USA).

\section{Cell culture}

T-47D breast cancer cell line was obtained from Iranian Biological Resource Center (IBRC, Tehran-Iran). T-47D was cultured in DMEM - Ham's F12 supplemented with $10 \% \mathrm{FBS}, 1 \%$ penicillin/streptomycin, at $37^{\circ} \mathrm{C}, 5 \%$ $\mathrm{CO} 2$ and humidified atmosphere. The cells were provided with fresh medium every 2 to 3 days. Cells were harvested at $80 \%$ confluence with trypsin $0.25 \%$ EDTA $0.02 \%$ and were either used fresh or frozen on liquid nitrogen (stored at $-70^{\circ} \mathrm{C}$ ). Shikonin was dissolved at a concentration of $50 \mathrm{mM}$ in DMSO as a stock solution and stored in the dark at $-20^{\circ} \mathrm{C}$. Nec-1 and Z-VAD-FMK were dissolved in DMSO to a storage concentration of $1 \mathrm{mM}$. Different concentrations $(\mu \mathrm{M})$ of shikonin, Nec-1and Z-VAD-FMK for cell line treatment were prepared in the cultured medium. Control cells were incubated with a volume of DMSO equal to that added to the cultures that received drugs. The maximum final concentration of DMSO was less than $0.1 \%$ for each treatment.

\section{Cell proliferation assay}

The assay is based on the reduction of yellow tetrazolium MTT (3-(4, 5-dimethylthiazolyl-2)-2, 5-diphenyltetrazolium bromide) by the alive cells (Salami and Karami-Tehrani, 2003). Briefly, adherent cells were detached by treatment with $0.25 \%$ trypsin/0.02\% EDTA and an aliquot of $7.5 \times 10^{3}$ cells were placed in each well $(200 \mu \mathrm{l})$ of 96-well plate (Thermo Scientific, Germany). Cells were allowed to attach overnight and then were stimulated with different concentrations of shikonin $(0.5$, $2.5,5,10,15,20,25 \mu \mathrm{M}$ ) for $6,12,24$ or 48 hours. Each well was incubated with $20 \mu \mathrm{l} \mathrm{MTT}$ at $37^{\circ} \mathrm{C}$ for $4 \mathrm{~h}$. The supernatant was then removed and $200 \mu 1$ DMSO was added into each well in order to solubilize the blue-purple crystals of formazan. Absorbance was measured at $570 \mathrm{~nm}$ with a microplate reader (TECAN, Austria). The viability was evaluated based on a comparison with untreated cells. $\mathrm{IC}_{50}$ values represent the shikonin concentrations required to inhibit $50 \%$ of cell proliferation and were calculated by GraphPad prism 6 (GraphPad Software, Inc. La Jolla, CA USA).

\section{Cell death assay}

To quantify the cell death modality, Annexin V/PI staining assay was applied. Briefly $3 \times 10^{5}$ cells were plated and pretreated with $50 \mu \mathrm{M} \mathrm{Nec}-1$ or $20 \mu \mathrm{MZ}$-VADFMK for 3 hours and then the cells were treated with shikonin ( $5 \mu \mathrm{M}$ for 12 hours). After treatment, cells were washed twice with PBS, mixed with $500 \mu$ l of binding buffer and stained with $5 \mu \mathrm{l}$ of Annexin V-FITC and $5 \mu \mathrm{l}$ of
PI for $10 \mathrm{~min}$ at room temperature in the dark. Apoptotic cells were quantified by a FACS Calibur flow cytometer (BD Biosciences, San Jose, CA). $1 \times 10^{4}$ cells were counted for each sample. Both early apoptotic (Annexin V-positive, PI-negative) and late apoptotic (double positive of Annexin V and PI) cells were detected. The percentages of cells in each quadrant were analyzed using flowing software version 2.4.1.

\section{Fluorescein Active Caspase-8 assay}

The fluorescent marker, FITC-IETD-FMK, is a labeled, cell-permeable, non-toxic inhibitor that binds irreversibly to activated caspase- 8 in living cells. Cultured Cells in 24-well plate were pretreated with $20 \mu \mathrm{M}$ Z-VADFMK or $50 \mu \mathrm{M} \mathrm{Nec}-1,3$ hours prior to treatment with 5 $\mu$ Mshikonin. After $24 \mathrm{~h}$ of treatment, according to the manufacturer's protocol, cellular active caspase- 8 was determined. Briefly, after treatment of cells by shikonin, $300 \mu \mathrm{l}$ each of the induced and control well Aliquot into eppendorf tubes. $1 \mu \mathrm{l}$ of FITC-IETD-FMK Added to each tube and incubate for 1 hour in a $37^{\circ} \mathrm{C}$ incubator with $5 \%$ $\mathrm{CO}_{2}$. Control cells were unlabeled. Cells centrifuged at $3000 \mathrm{rpm}$ for 5 minutes and removed supernatant. Cells resuspended in $0.5 \mathrm{ml}$ of wash buffer, and centrifuged again. Cells resuspended in $100 \mu$ of wash buffer then the cell suspension transferred to each well of the black microtiter plate and the fluorescence intensity measured at excitation $485 \mathrm{~nm}$ and emission $535 \mathrm{~nm}$.

\section{Caspase-3 activity assay}

The influence of shikonin on caspase- 3 activity in T-47D was detected using Caspase-3/CPP32 Fluorometric Assay Kit (BioVision Research Products, Mountain View, CA, USA). Cleavage of DEVD-AFC (AFC: 7-amino4-trifluoromethyl coumarin) was detected for caspase 3 activity. DEVD-AFC emits blue light $(\lambda \max =400 \mathrm{~nm})$ but free AFC, upon cleavage of the substrate by CPP32, emits yellow-green $(\lambda \max =505 \mathrm{~nm})$. Cultured Cells in 96-well plate, were pretreated with $20 \mu \mathrm{MZ}$-VAD-FMK or $50 \mu \mathrm{M}$ Nec-1, 3 hours prior to treatment with $5 \mu \mathrm{M}$ shikonin. After $12 \mathrm{~h}$ of treatment, $0.5-2 \times 10^{5}$ cells were lysed in $50 \mu \mathrm{l}$ chilled Cell lysis buffer on ice, for 10 minutes. The amount of protein in each well was measured using the Bradford method (Stoscheck, 1990). After addition of $50 \mu \mathrm{l}$ of $2 \mathrm{X}$ reaction buffer (containing $10 \mathrm{mM}$ DTT), Cell lysate from shikonin treated or control cells were incubated at $37^{\circ} \mathrm{C}$ for $2 \mathrm{~h}$ with $50 \mu \mathrm{M}$ DEVD-AFC substrate. Samples were read at $400 \mathrm{~nm}$ excitation filter and $505 \mathrm{~nm}$ emission filter with a fluorescent micro plate reader (BioTek Synergy Ht, Winooski, Vermont, USA) and results were expressed as fold increase over the basal level (control cells).

\section{Intracellular reactive oxygen species assay}

For the assessment of intracellular ROS formation in cultured breast cancer cells, a fluorescent probe 2', $7^{\prime}$-dichlorofluorescin diacetate (DCFH-DA) was used. DCFH-DA was dissolved in PBS at a concentration of 10 $\mathrm{mM}$. To assay ROS, cells were plated in 96 cell well plate. Assay was administered with DCFH-DA $(20 \mu \mathrm{M}$ working substrate solution) in culture medium. After incubation at $37^{\circ} \mathrm{C}$ for $45 \mathrm{~min}$, substrate solution was removed and 
Shikonin Induced Necroptosis Via Reactive Oxygen Species in T-47D Breast Cancer Cells

cells were washed with PBS. Shikonin added to wells at concentration $5 \mu \mathrm{M}$ to generate ROS for 4 Hours. Untreated cells were used as control. Fluorescence was measured at an excitation wavelength of $485 \mathrm{~nm}$ and an emission wavelength $528 \mathrm{~nm}$ using a fluorescent micro plate reader (BioTek Synergy Ht, Winooski, Vermont, USA). The ROS levels were expressed as RFU (Relative Fluorescence Unit).

\section{Statistical analysis}

$\mathrm{IC}_{50}$ value was determined using GraphPad Prism statistical software 6 (CA, USA).To compare the data,<smiles>CC(C)=CCC(O)C1=CC(=O)c2c(O)ccc(O)c2C1=O</smiles>

Figure 1. Structure of shikonin
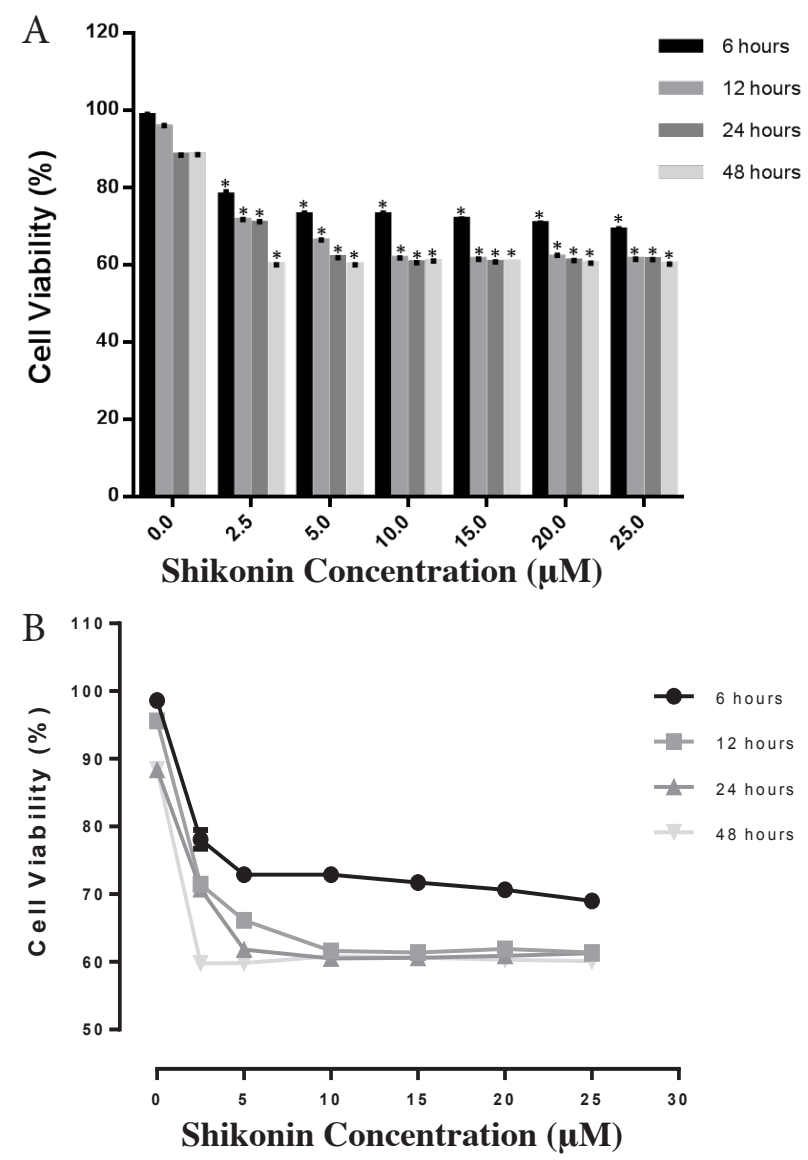

Figure 2. shikonin Induces Cell Death in T-47D Cell Line in the dose and time Dependent Manner. A) T-47D cells were treated with shikonin in the increasing concentrations $(2.5,5,10,15,20$ and $25 \mu \mathrm{M})$ for $6,12,24$ or 48 hours as indicated. B) Inhibition trend of cell proliferation by shikonin. Data shown are mean \pm SD of triplicate samples at four independent experiments with similar results. ***p < 0.001 denote mean significantly different from untreated cells unpaired t-test and one-way analysis of variance (ANOVA) followed by Dunnett's post-hoc test. In all cases, the mean \pm S.D of at least 3 independent experiments were presented; $\mathrm{P}<0.05$ was taken as the level of significance.

\section{Results}

Shikonin inhibits T-47D cells proliferation in a dose-and time-dependent manner

As shown in Figure 2A, shikonin significantly inhibited the proliferation of T-47D cells in a time- and dose-dependent manner. Shikonin treatment resulted in a dose dependent reduction in cell proliferation when compared with control cells. The cytotoxic effect was more evident at $48 \mathrm{~h}$ in cell line. The effective dose of shikonin that inhibited $50 \%$ of growth $\left(\mathrm{IC}_{50}\right)$ of T-47D cells after $12 \mathrm{~h}$ of treatment were $3.586 \mu \mathrm{M}$, respectively.
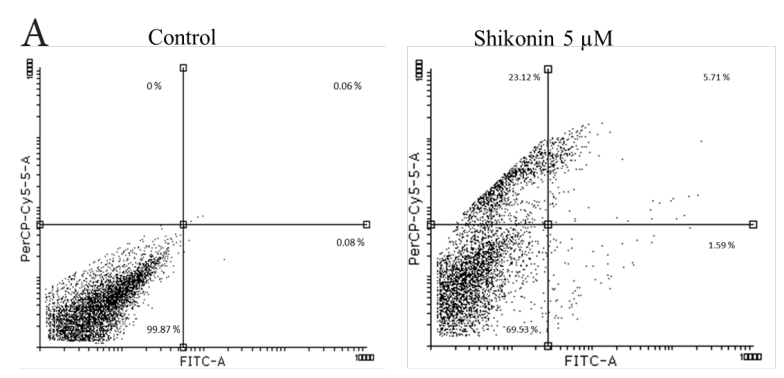

Shikonin $5 \mu \mathrm{M} / \mathrm{Z}$-VAD $20 \mu \mathrm{M}$

Shikonin $5 \mu \mathrm{M} / \mathrm{Nec}-150 \mu \mathrm{M}$
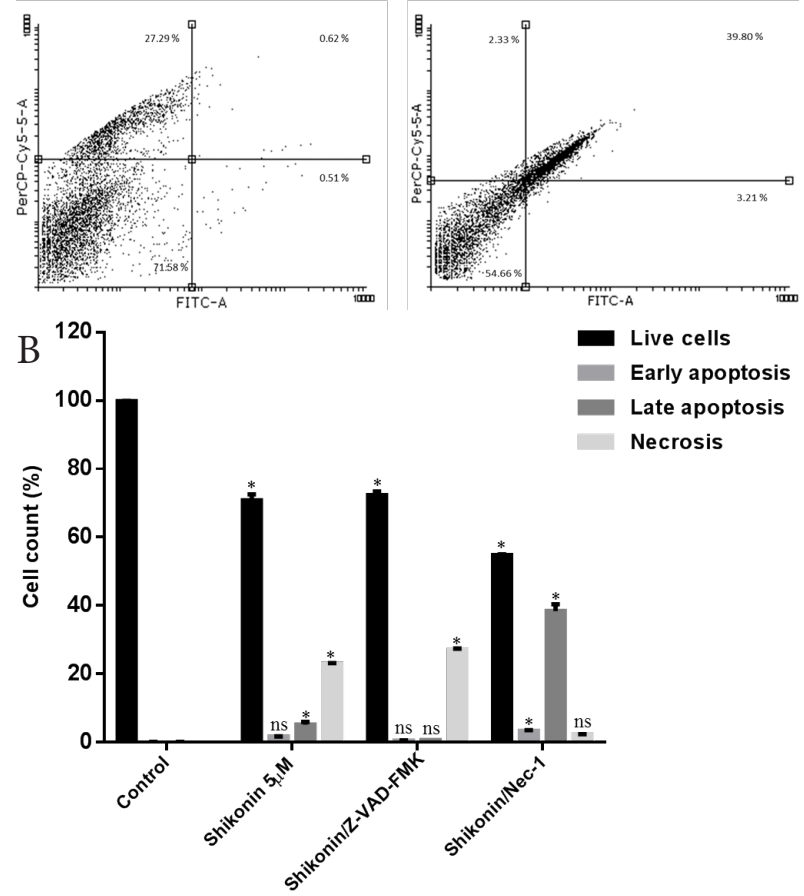

Figure 3. Shikonin Induced Cell Modes in T-47D cell line. A) shikonin $(5 \mu \mathrm{M})$ treated T-47D cells in the absence or presence of Nec-1 $(50 \mu \mathrm{M})$ and Z-VAD-FMK $(20 \mu \mathrm{M})$ for 12 hours. Nec-1 and Z-VAD-FMK were pretreated for 3 hours prior to shikonin treatment. B) After 12 hours incubation with shikonin, both the percentages of necrotic cells (stained with PI only) and late apoptotic cells (stained with both Annexin V and PI) increased significantly. The necrotic cells were significantly inhibited by pretreatment with Nec-1 and early and late apoptotic cells suppressed by pre-incubation with Z-VAD-FMK. Data are representative of 3 independent experiments. ${ }^{*} p<0.001$ denote mean significantly different from untreated cells. 


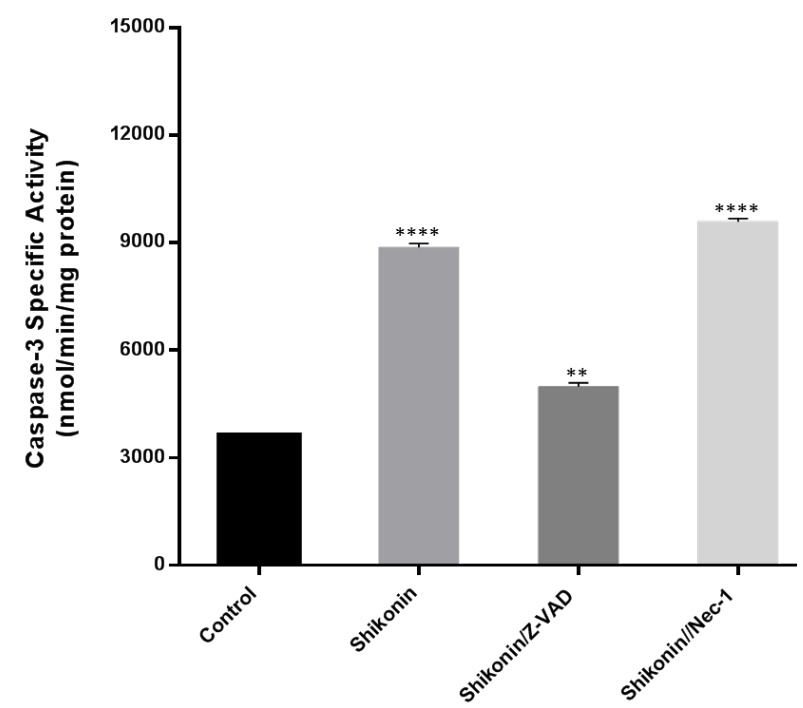

Figure 4. Detection of active caspase- 8 in the presence of shikonin in T-47D cells. The cells were treated with shikonin at the final concentration of $5 \mu \mathrm{M}$ in the absence or presence of Nec-1 $(50 \mu \mathrm{M})$ and Z-VAD-FMK $(20 \mu \mathrm{M})$ for 24 hours while the untreated cells were used as control. Nec-1 and Z-VAD-FMK were pretreated for 3 hours before shikonin treatment. $* * * * p<0.0001$ denote mean significantly different from untreated cells

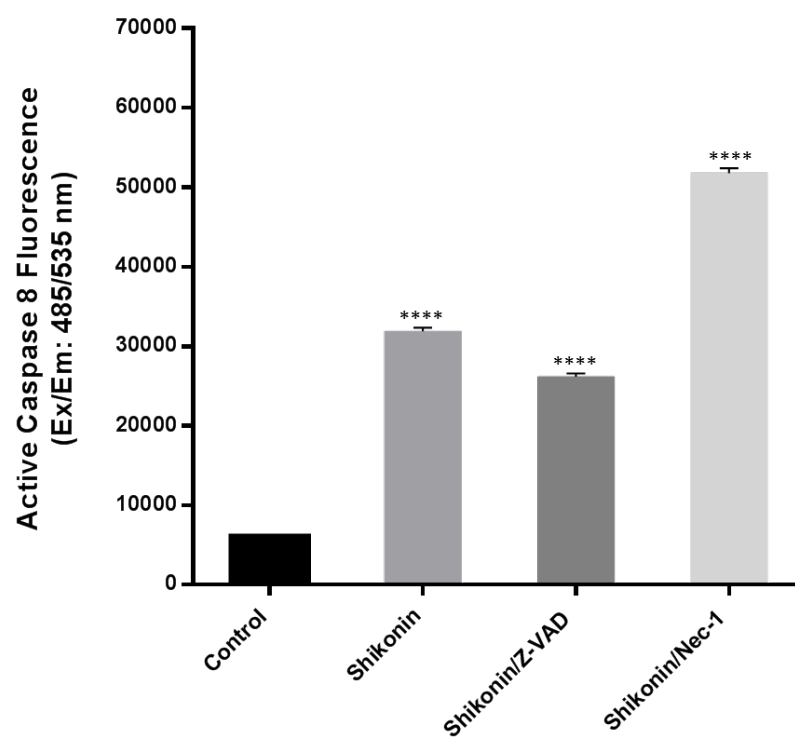

Figure 5. shikonin induces a caspase-3 dependent apoptosis in T-47D cells. The activity of caspase- 3 is increased after shikonin treatment. Z-VAD-FMK decreased caspase- 3 specific activity and Nec-1 increased that. The amount of protein was measured using the Bradford method. ****p < 0.0001 denote mean significantly different from untreated cells, ns: not significant different from untreated cells.

Concentration of $5 \mu \mathrm{M}$ used as optimum concentration in the subsequent experiments (Figure 2B). Therefore, T-47D cells are exquisitely sensitive to cell death induced by shikonin treatment.

Shikonin induces both apoptosis and necroptosis in T-47D breast Cancer Cells

In order to examine the effects of shikonin on the cells, FITC-conjugated annexin V and PI staining were

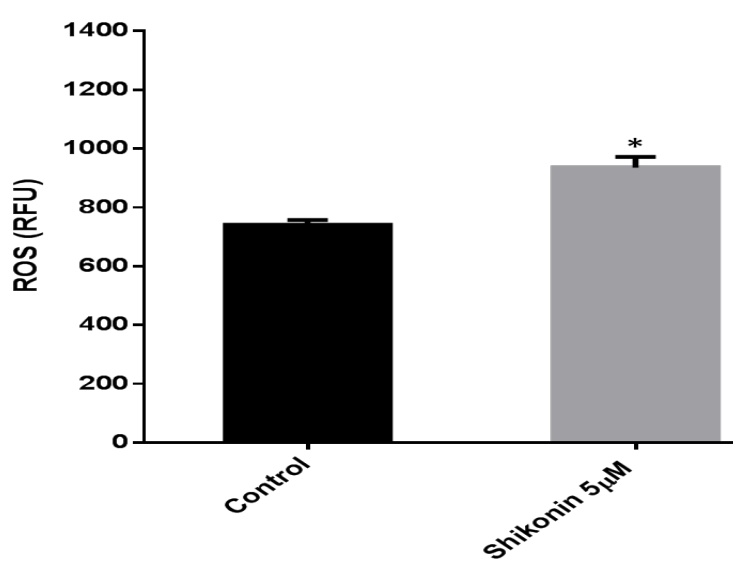

Figure 6. Shikonin mediates ROS production in T-47D breast cancer cells. Cells were loaded with 2', 7 '-Dichlorofluorescin diacetate at $20 \mu \mathrm{M}$ final concentration for 45 minutes before treating with shikonin $(5 \mu \mathrm{M})$ for 4 hours. Data points represent RFU (Relative Fluorescence Unit) of three independent experiments. ${ }^{* *} \mathrm{p}<0.01$ denote mean significantly different from untreated cells.

used. Cells were incubated with shikonin, $5 \mu \mathrm{M}$, for 12 h. Control cells were negative for both annexin V-FITC and PI (Figure 3A). A significant increase has been shown in the percentage of apoptosis in $\mathrm{T}-47 \mathrm{D}$ cells, $(\mathrm{P} \leq 0.05)$ (Figure 3B.). Necroptosis is optimally induced when the apoptotic machinery is interrupted. To delineate the cell death modes by shikonin, T-47D cells were treated by shikonin in combination with either Z-VAD-FMK or Nec-1. Taken together, shikonin induces a dominant necroptosis in T-47D cells. Importantly, this shikonininduced necroptosis observed was inhibited in the presence of the RIP1K kinase inhibitor Nec-1. Nec-1 converts shikonin induced necrosis to apoptosis. The addition of Z-VAD-FMK to cells under apoptosis-inducing condition, reduced apoptosis. No significant alteration was found in the shikonin and shikonin/Z-VAD-FMK treated cells at early apoptosis stage in compared to control cells, but early apoptosis stage of shikonin/Nec-1 treated cells increased significantly. Similarly, the cells at late apoptosis stage were also attenuated by Z-VAD-FMK. There was no significant difference between percentages of necrosis in shikonin/Nec-1 treated cells with control cells. This suggested that Nec-1 effectively blocked shikonin induced necrosis.

Involvement of caspase-3 and 8 in shikonin induced apoptosis

The results demonstrated that treatment of T-47D cells with $5 \mu \mathrm{M}$ of shikonin resulted in a significant increase $(\mathrm{P}<0.01$, compared with control) in the activity of caspase-8 (Figure 4) and caspase-3 (Figure 5). Further confirmation for the involvement of caspases in the induction of apoptosis by shikonin was provided by the results obtained from pretreatment of the cells with Z-VAD-FMK $(20 \mu \mathrm{M})$, a broad-spectrum caspase inhibitor. Z-VAD-FMK significantly $(\mathrm{P}<0.01$, compared with control) inhibited shikonin -induced apoptosis in $\mathrm{T}-47 \mathrm{D}$ cells. Here we showed that shikonin in the presence 
of Nec-1 $(50 \mu \mathrm{M})$ increased caspase-3 and 8 activity. Therefore, these results suggest that Nec-1 also converts necroptosis into apoptosis in the breast cancer cells and shikonin induced apoptosis in breast cancer cell line is caspase dependent.

\section{ROS involvement in shikonin-induced cell death}

After treatment with shikonin, the intracellular ROS was determined by the DCFH-DA probe. In T-47D cells, after treatment by shikonin, a significant increase was observed in ROS levels when compared with those of control cells (shown in Figure 6). Exposure of cells to shikonin led to mitochondrial dysfunction that resulted in ROS production, which contributed to necroptosis. We observed increased ROS production shortly after cellular shikonin uptake, and ROS levels continuously increased for at least $1 \mathrm{~h}$ after exposure to shikonin. Thus, these data indicated that shikonin induced necroptosis was associated as well with oxidative stress. Thus, shikonin is indeed a potent ROS inducer.

\section{Discussion}

Induction of necroptosis may improve the effect of anticancer drugs, especially those of drug-resistant cancers (Xun $\mathrm{Hu}, 2007$ ). Therefore, in the present study we assessed the effects of shikonin on the induction of two programmed cell death pathways, apoptosis and necroptosis, in the breast cancer cell line, T-47D. Our results revealed that shikonin disrupts the breast cancer cells line, either by necroptosis or apoptosis. While apoptotic pathway was blocked by Z-VAD-FMK, necroptosis became dominant and when necroptotic pathway was inhibited by Nec-1, apoptosis was a selective route for the cell death. Although shikonin has induced both routs of cell death, indeed it is a potent necroptotic inducer.

Caspase- 3 activation is responsible for the final step of apoptosis (Siao et al., 2015). In the present investigation, shikonin induced a typical caspase-3 dependent apoptosis in T-47D cell line. Induction of caspase dependent apoptosis by shikonin has also been reported in other malignant cell (Fu et al., 2013; Huang et al., 2013).

ROS are very important in mediating cell necrosis via death receptor such as TNFR1 (Park et al., 2013). In the hepatoma SK-Hep-1 cells, shikonin has produced large quantities of intracellular ROS in the early stage of apoptosis which was then followed by the disruption of mitochondrial transmembrane potential (Jen-Tsung Yang, 2014). Intracellular ROS elevation and mitochondrial destruction may result in the oxidative DNA damage, suppression of cancer cell migration and cell cycle arrest (Wiench et al., 2012; Wang and Zhang, 2015). In order to achieve therapeutic selectivity and to overcome drug resistance, targeting this biochemical change might be useful. Using ROS-generating agents together with ROS elimination inhibitors resulted in the accumulation of ROS and increase in the cancer cell cytotoxicity (Wang and Zhang, 2015). In the present study, parallel with the results of other investigations, we have observed an increase in the levels of ROS in the presence of shikonin, as a potent ROS inducer.

In conclusion, the current study has indicated that shikonin mainly induces necroptosis. However, in the presence of Nec-1, caspase-dependent apoptosis has been occurred. Shikonin stimulates ROS generation in the mitochondria of T-47D cells, which causes necroptosis or apoptosis. However induction of necroptosis, as a backup programmed cell death pathway via ROS stimulation, is a new strategy for the treatment of breast cancer.

\section{Acknowledgements}

Part of this work was supported by a Ph.D. grant from Tarbiat Modares University.

\section{References}

Baghestani AR, Shahmirzalou P, Zayeri F, et al (2015). Prognostic factors for survival in patients with breast cancer referred to omitted cancer research center in Iran. Asian Pac J Cancer Prev, 16, 5081-4.

Christofferson DE LY, Yuan J (2014). Control of life-or-death decisions by RIP1 kinase. Annu Rev Physiol, 76, 129-50.

Christofferson DE (2010). Necroptosis as an alternative form of programmed cell death. Curr Opin Cell Biol, 22, 263-8.

Doval DC SA, Sinha R, Kumar K, et al (2015). Immunohistochemical profile of breast cancer patients at a tertiary care hospital in new delhi, India. Asian Pac J Cancer Prev, 16, 4959-64.

Fu Z DB, Liao Y, Shan L, et al (2013). The anti-tumor effect of shikonin on osteosarcoma by inducing RIP1 and RIP3 dependent necroptosis. BMC Cancer, 13, 580.

Ghavami S HM, Ande SR, Yeganeh B, et al (2009). Apoptosis and cancer: mutations within caspase genes. J Med Genet, 46, 497-510.

Huang C LY, Zhao J, Yang F, et al (2013). Shikonin kills glioma cells through necroptosis mediated by RIP-1. PLoS One, $\mathbf{8 ,} 66326$.

Jen-Tsung Yang Z-LL, Jin-Yi Wu, Fung-Jou Lu, Ching-Hsein Chen (2014). An oxidative stress mechanism of shikonin in human glioma cells. PLOS ONE, 9, 1-12.

Mansoori AA (2015). Molecular links between alcohol and tobacco induced dna damage, gene polymorphisms and patho-physiological consequences: a systematic review of hepatic carcinogenesis. Asian Pac J Cancer Prev, 16, 4803-12.

Meka PB JS, Nanchari SR, Vishwakarma SK, et al (2015). LCN2 promoter methylation status as novel predictive marker for microvessel density and aggressive tumor phenotype in breast cancer patients. Asian Pac J Cancer Prev, 16, 4965-9.

Park S, Cho Y (2013). Shikonin induces programmed necrosislike cell death through the formation of receptor interacting protein 1 and 3 complex. Food Chem Toxicol, 55, 36-41.

Sadeghi RN, Karami-Tehrani F, Salami S (2015). Targeting prostate cancer cell metabolism: impact of hexokinase and CPT-1 enzymes. Tumour Biol, 36, 2893-905.

Salami S, Karami-Tehrani F (2003). Biochemical studies of apoptosis induced by tamoxifen in estrogen receptor positive and negative breast cancer cell lines. Clin Biochem, 36, 247-53.

Sharifian A PM, Emadedin M, Rostami Nejad M, et al (2015). Burden of breast cancer in iranian women is increasing, Asian Pac J Cancer Prev, 16, 5049-52.

Shindo R, Okumura K, Kumagai Y, Nakano H (2013). Critical contribution of oxidative stress to TNFa-induced necroptosis 
downstream of RIPK1 activation. Biochem Biophys Res Commun, 436, 212-6.

Siao AC, Hou CW, Kao YH, et al (2015). Effect of sesamin on apoptosis and cell cycle arrest in human breast cancer mcf-7 cells. Asian Pac J Cancer Prev, 16, 3779-83.

Singha PK, Pandeswara S, Venkatachalam MA, et al (2013). Manumycin A inhibits triple-negative breast cancer growth through LC3-mediated cytoplasmic vacuolation death. Cell Death Dis, 4, 457.

Stoscheck CM (1990). Quantitation of protein. Methods Enzymol, 182, 50-68.

Tavakoli-Yaraki M, Karami-Tehrani F, Salimi V, et al (2013). Induction of apoptosis by Trichostatin A in human breast cancer cell lines: involvement of 15-Lox-1. Tumour Biol, 34, 241-9.

Wang HY, Zhang B (2015). Cobalt chloride induces necroptosis in human colon cancer HT-29 cells. Asian Pac J Cancer Prev, 16, 2569-74.

Wiench B ET, Paulsen M, Efferth T (2012). Shikonin directly targets mitochondria and causes mitochondrial dysfunction in cancer cells. Evid Based Complement Alternat Med, 2012, 726025.

Wu H XJ, Pan Q, Wang B, Hu D, Hu X (2013). Anticancer Agent Shikonin Is an Incompetent Inducer of Cancer Drug Resistance. PLoS One, 8, 52706.

Xin Y, Li XY, Sun SR, et al (2015). Vegetable oil intake and breast cancer risk: a meta-analysis. Asian Pac J Cancer Prev, 16, 5125-35.

Xun Hu WHLL (2007). Targeting the weak point of cancer by induction of necroptosis. Autophagy, 3, 490-2. 